\title{
Galactose Oxidase Enables Modular Assembly of Conjugates from Native Antibodies with High Drug-to-Antibody Ratios
}

\author{
Antonio Angelastro, ${ }^{[a]}$ Alexey Barkhanskiy, ${ }^{[a]}$ Ashley P. Mattey, ${ }^{[a]}$ Edward G. Pallister, ${ }^{[a]}$ Reynard \\ Spiess, ${ }^{[a]}$ William Goundry, ${ }^{[b]}$ Perdita Barran, ${ }^{[a]}$ and Sabine L. Flitsch ${ }^{[a]},{ }^{*}$
}

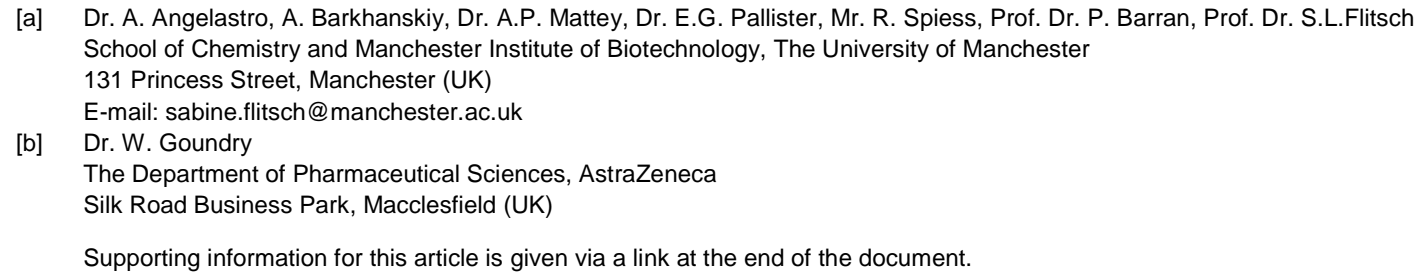

\begin{abstract}
The potential of antibody conjugates with high drug loading in anticancer therapy has recently been highlighted by the approval of Trastuzumab deruxtecan and Sacituzumab govitecan. These biopharmaceutical approaches have spurred interest in bioconjugation strategies with high and defined degrees antibody-todrug (DAR) ratios, in particular on native antibodies. Here we report a glycoengineering methodology to generate antibody drug conjugates with DAR of up to eight, by combining highly selective enzymatic galactosylation and oxidation with biorthogonal tandem KnoevenagelMichael addition chemistry. This three step approach offers a selective route to conjugates from native antibodies with high drug loading, and thus illustrates how biocatalysis can be used for the generation of biopharmaceuticals using mild reaction conditions.
\end{abstract}

Antibody-drug conjugates (ADCs), the combination between the cancer-targeting capabilities of monoclonal antibodies (mAbs) and cancer-killing ability of cytotoxic drugs, are highly regarded in biotherapeutics. ${ }^{[1]}$ Antibody-to-drug ratio (DAR), the average number of payloads linked to each antibody, is a crucial attribute in developing an ADC from bench to clinic. ${ }^{[2]}$ While cytotoxicity and thus potency - is expected to increase linearly with drug loading, the average DAR for most of clinically approved ADCs ranges between 2 and 4 , which is commonly accepted as the best compromise in obtaining conjugates with favourable therapeutic index. ${ }^{[2,4]}$ Nevertheless, the recent approval of Trastuzumab deruxtecan (Enhertu, DAR 7.7) ${ }^{[5]}$ and Sacituzumab govitecan (Trovedly, DAR 7.6 ${ }^{[6]}$ prompted a re-evaluation of high- over low-to-moderate DAR ADCs. ${ }^{[4 b, 7]}$ The majority of conjugation strategies involve chemical cysteine modification. ${ }^{[4 b, 7]}$ Such approaches can perturb the covalent linkages between the heavy and light chains. ${ }^{[8]}$

With the advancement of site-specific conjugation techniques ${ }^{[9]}$ it has become evident that ADCs with precise and homogeneous payload distribution generally show improved pharmacological properties in respect to their mixed load counterparts. ${ }^{[3,10]}$ However, compared to low-to-moderate DAR ADCs, ${ }^{[3,}$, 11] methodologies for high-DAR conjugates synthesis are limited; ${ }^{[10}$, ${ }^{12]}$ this issue is remarkably tangible when native antibodies are considered. [4b, 5a, 6a, 11d-e, 12]

In this work, we describe an alternative route to the site-specific production of high-DAR ADCs by targeting the $\mathrm{N}$-glycan chains of Trastuzumab 1 and using biocatalysis as a mild method for introduction of biorthogonal groups. Key steps involve the enzymatic oxidation of the C6-hydroxyl group of D-galactose into C6-aldehyde by galactose oxidase (GOase, Figure 1 \& 2), ${ }^{[13]}$ followed by ligation to each aldehyde of two linker-payload via tandem Knoevenagel-Michael addition (TKM, Figure 3). ${ }^{[4 c]}$

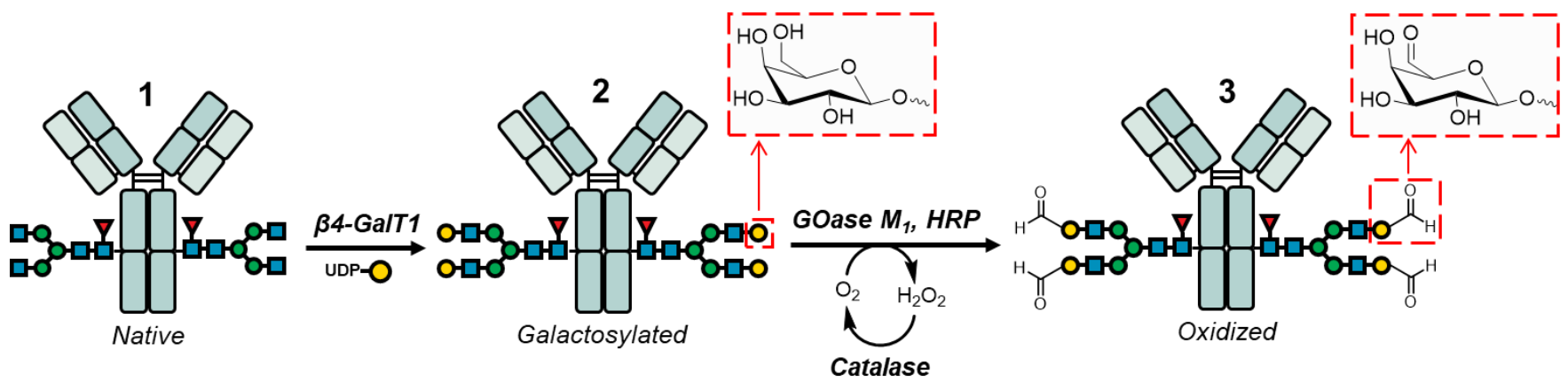

Figure 1. Glycoengineering strategy to introduce four aldehyde groups into Trastuzumab 1 for subsequent site-specific functionalization. In the first biocatalytic step, $\beta$-1,4-galactosyltransferase 1 ( $\beta 4$-GalT1) transfers D-galactose units from UDP-Gal to each biantennary N-glycan chain of native Trastuzumab 1. Subsequently, C6-hydroxyl groups of galactose units are oxidized to aldehyde with galactose oxidase (GOase $\left.\mathrm{M}_{1}\right)$. 
During the first biocatalytic step, G0F, the dominant N-glycoform of native trastuzumab 1, is galactosylated into G2F 2 using $\beta-1,4$ galactosyltransferase 1 ( $\beta 4-G a l T 1$ ) and UDP-Gal as sugar donor (Figure 1, 2a,b). ${ }^{[14]}$ Subsequently, GOase $M_{1}$, an engineered variant that can be rapidly produced in $E$. coli, ${ }^{[15]}$ was employed to oxidize each galactose unit of the $\mathrm{N}$-glycan into galactoaldehydes $3 .^{[13]}$ The oxidized Trastuzumab 3 was analysed after fragmentation by either Trypsin to generate glycopeptides or by selective proteolytic digestion using IdeZ protease to generate intact $\mathrm{Fc} / 2$ fragments. The fragments were analysed by two independent and complementary techniques, i.e. HILIC/MS for the corresponding tryptic digest and RPLC/MS for the $\mathrm{Fc} / 2$ fragments (Figure 2). These were in agreement confirming that GOF sidechains were galactosylated (Figure $2 \mathrm{~b}$ ) and oxidized (Figure 2c) in a highly efficient manner under optimized conditions (supporting information).

Although the HILIC chromatogram showed single product peaks, multiple $\mathrm{m} / \mathrm{z}$ peaks were observed in the mass spectrum of oxidised Fc fragment of $\mathbf{3}$, which we assigned as different hydration states of the aldehydes (Figure 3c). To confirm the identity of the tetra-aldehyde $\mathbf{3}$, oxidized Trastuzumab $\mathbf{3}$ was subjected to reduction with sodium borodeuteride, and the resulting tryptic digest analysed by HILIC/MS (Figure 2d). Owing to borodeuteride reduction of aldehydes, conversion of G2F galactoaldehydes into C6-deuterated galactose $\left(\left[^{2} \mathrm{H}_{2}\right]-\mathrm{G} 2 \mathrm{~F}\right)$ resulted in mass spectra that could easily be assigned to the proposed deuteriated product $\left[{ }^{2} \mathrm{H}_{4}\right]-3$ and suggested that the previous oxidation was clean.

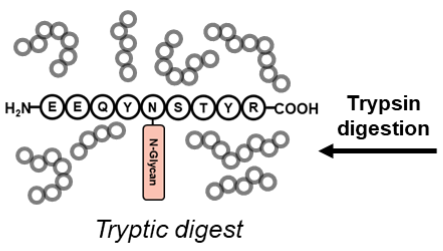

HILIC Chromatogram

a) Native 1

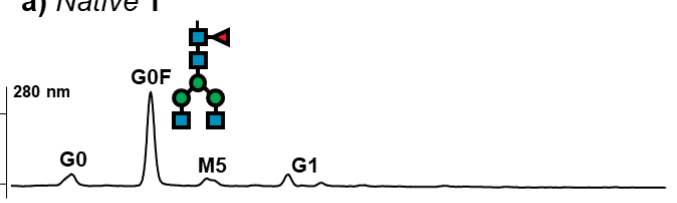

b) Galactosylated 2

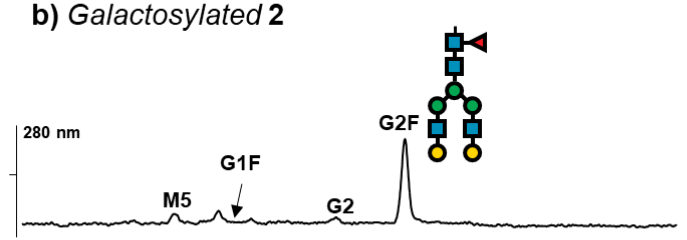

c) Oxidized 3

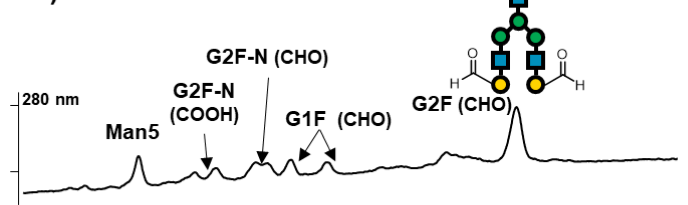

d) Deuteriated $\left[{ }^{2} \mathrm{H}_{4}\right]-3$

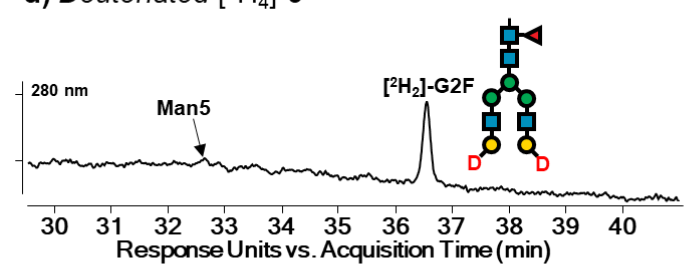

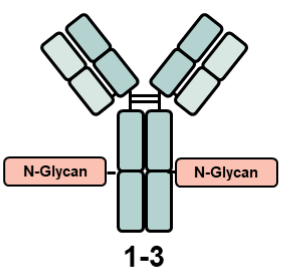

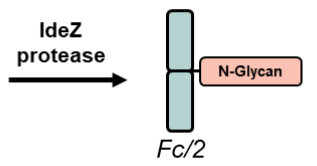

Fc/2 Fragment
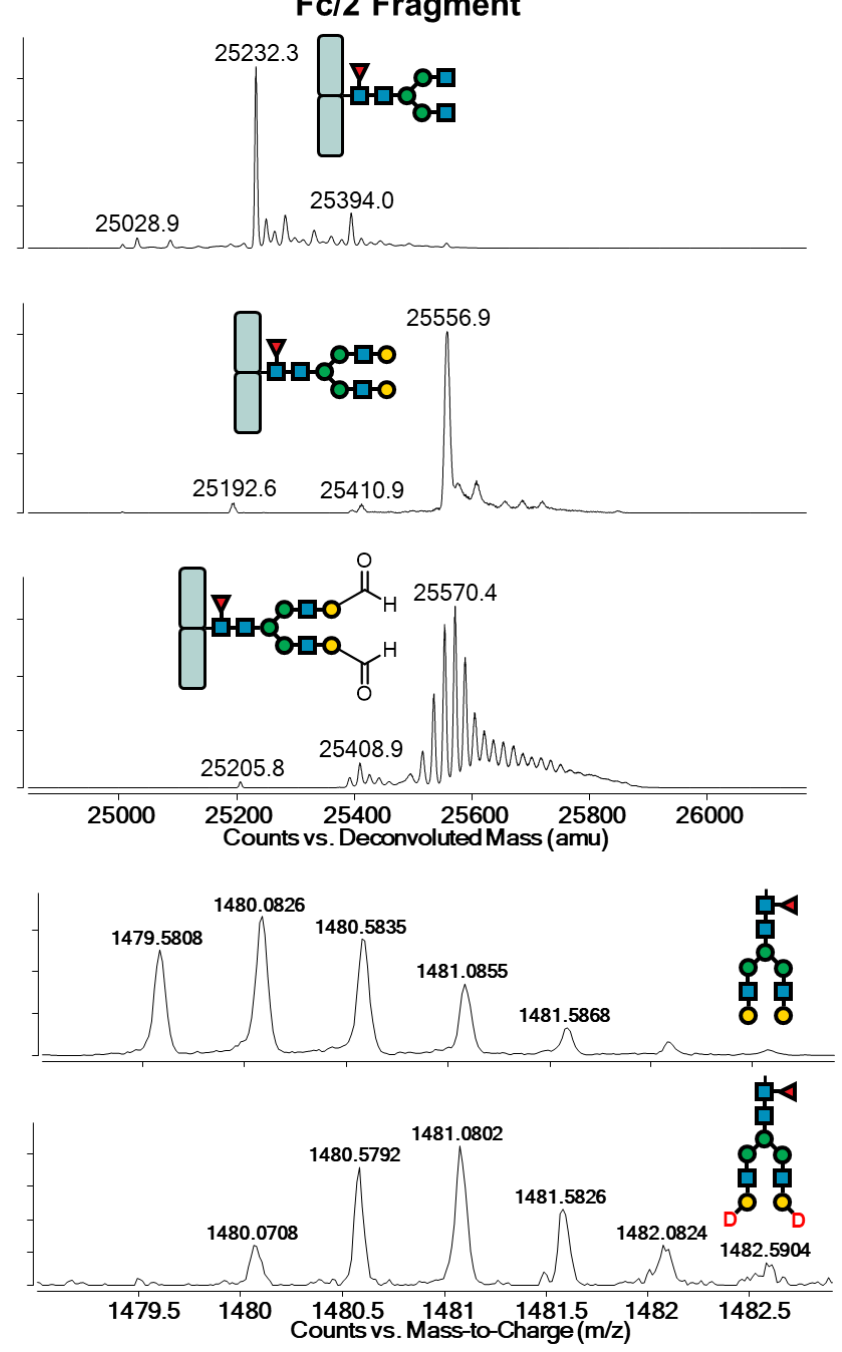

Figure 2. HILIC/MS and Fc/2 analyses of N-glycosylation patterns of a) native 1, b) galactosylated 2 and c) oxidized $\mathbf{3}$ Trastuzumab. d) HILIC/MS analysis of GOase-oxidized Trastuzumab $\left[{ }^{2} \mathrm{H}_{4}\right]-3$ after reduction of $\mathbf{3}$ with sodium borodeuteride. See supporting information for details (Figures $\mathrm{S} 1$-S19 \& S20-S23) and experimental procedures. 
Having optimized the enzymatic steps to introduce four aldehyde biorthogonal handles on the $\mathrm{N}$-glycan chain of $\mathbf{1}$, we next focused on linker-payload attachment via the TKM reaction (Figure 3a). TKM ligation has been employed in ADC technologies combined to formylglycine-generating enzyme (FGE), ${ }^{[16]}$ which catalyzes the oxidation of an engineered cysteine residue within a consensus sequence into aldehyde, to produce DAR $\sim 4$ ADCs. ${ }^{[4 c,}$ 17] Compared to the FGE/TKM strategy, our GOase mediated Nglycan oxidation would work on native antibodies and not require antibody re-engineering. In addition, our approach would access high DAR numbers of up to 8 from the tetra-aldehyde 3.

As proof-of-principle eight azido groups were installed on oxidized trastuzumab 3 upon reaction with a bifunctional azido-pyrazolone linker 4 (Figure 3a) to generate azido-trastuzumab $\mathbf{5}$. Analysis of the $\mathrm{Fc} / 2$ fragment of $\mathbf{5}$ by mass spectrometry clearly showed a major peak at 26971 amu in agreement with the proposed structure and one lower DAR product at 26279 amu as a minor impurity.

The Fc/2 fragment of 5 was further analyzed after deglycosylation with PNGase F (Figure 3c) which shows native mass of 23787 amu and demonstrates that the TKM reaction is highly selective for the $\mathrm{N}$-glycan chain without alterations of the protein backbone. Analysis of deconvoluted MS data of the $\mathrm{Fc} / 2$ fragment generated from 5 (Figure S28) indicates G2F decorated with four azido groups being the dominant form (90\%), while $\mathrm{N}$-glycans partially functionalized with two azido moieties represent $2 \%$ of the observed species. The remaining species not being subjected to TKM conjugation were estimated $8 \%$. Overall, the approximate average number of azido groups available for conjugation (and thus the theoretical DAR) is 7.3 (see supporting information for DAR estimation).

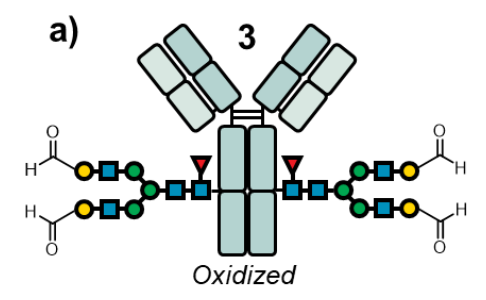

Tandem KnoevenagelMichael Addition (TKM)
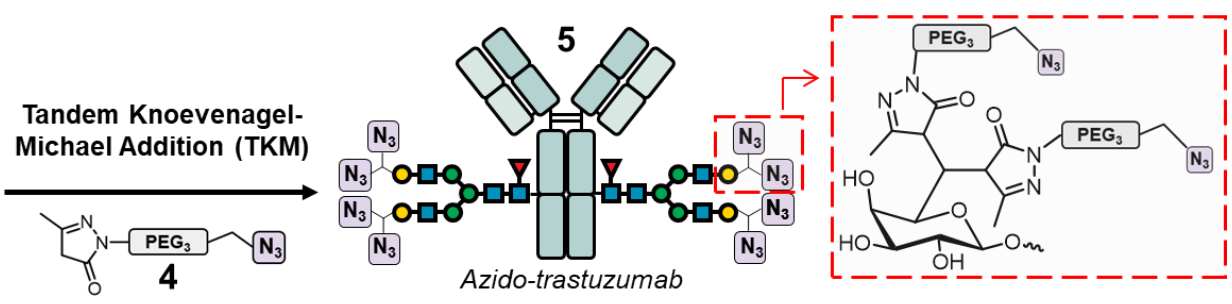

b)
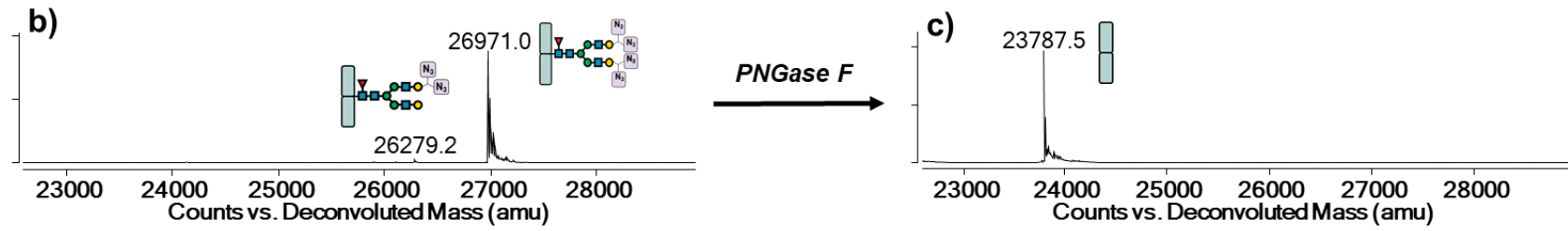

Figure 3. a) Functionalization of oxidized Trastuzumab 3 with 8 azido groups via Tandem Knoevenagel-Michael addition (TKM). The bifunctional azido-pyrazolone linker $\mathbf{4}$ was synthesised in a single step from commercially available starting material (supporting information). Fc/2 analysis of the functionalized antibody $\mathbf{5}$ b) before and c) after treatment with PNGase F shows functionalization of the oxidized N-glycan chain with four azido-pyrazolone linkers being specific.

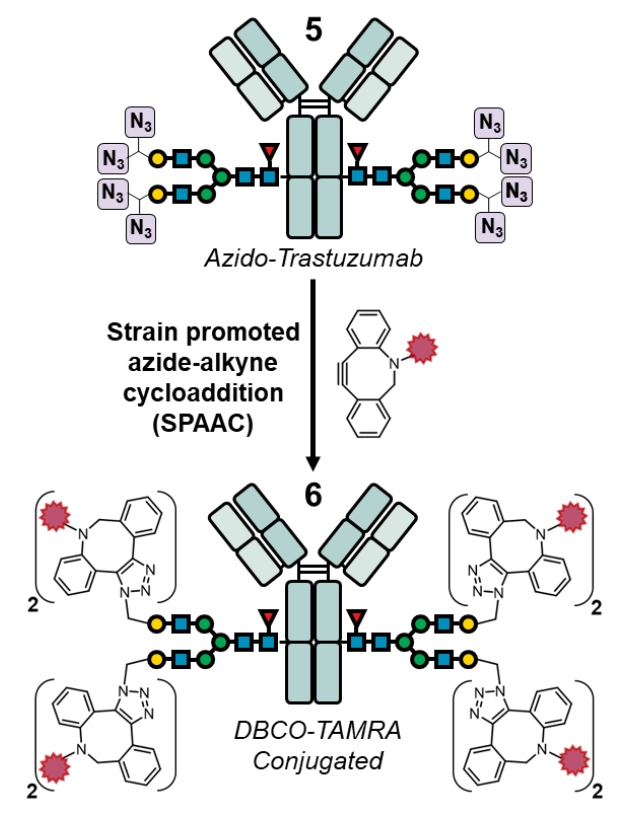

Figure 4. Conjugation of azido-Trastuzumab 5 to DBCO-TAMRA via by Strainpromoted azide-alkyne cycloaddition (SPAAC).
A mAb functionalized with up to eight azido moieties provides the advantage to rapidly assemble various high-DAR ADCs via e.g. strain-promoted azide-alkyne cycloaddition (SPAAC) using different payloads. ${ }^{[18]}$ We exemplified this by reacting azidotrastuzumab 5 with DBCO-TAMRA to generate the corresponding conjugated antibody 6 (Figure 4 \& supporting information). Accordingly, RPLC/MS analysis of the Fc/2 fragment generated from 6 shows the N-glycan conjugated to four DBCO-TAMRA being the dominant species (Figure S27 \& S28).

In summary, by combining biocatalysis with TKM chemistry, we have developed a glycoengineering methodology to produce ADCs with a molecular DAR of up to 8 and an average DAR of 7.3. As proof of concept, we have synthesized Trastuzumab functionalized with up to eight azido groups, which could be in turn readily conjugated to different payloads via click chemistry. This enzymatic methodology involving glycosyltransferases and oxidases represents a mild and selective alternative to chemical methods in synthesizing homogeneous high-DAR ADCs from native antibodies. 


\section{Acknowledgements}

We are very grateful to Dr. R. James Christie for providing us Trastuzumab and SYNBIOCHEM for MS facilities. This work was supported by the EPSRC, BBSRC and AstraZeneca (EP/S005226/1, BB/M017702/1).

Keywords: Antibody-drug conjugates - Biocatalysis • Glycoengineering

[1] a) P. Khongorzul, C. J. Ling, F. U. Khan, A. U. Ihsan, J. Zhang, Mol. Cancer Res. 2020, 18, 3-19; b) S. Baah, M. Laws, K. M. Rahman, Molecules 2021, 26, 2943.

[2] a) K. J. Hamblett, Clin. Cancer Res. 2004, 10, 7063-7070; b) E. Jabbour, S. Paul, H. Kantarjian, Nat. Rev. Clin. Oncol. 2021, 18, 418-433; c) A. T. Lucas, L. S. L. Price, A. N. Schorzman, M. Storrie, J. A. Piscitelli, J. Razo, W. C. Zamboni, Antibodies 2018, 7, 10; d) X. Sun, J. F. Ponte, N. C. Yoder, R. Laleau, J. Coccia, L. Lanieri, Q. Qiu, R. Wu, E. Hong, M. Bogalhas, L. Wang, L. Dong, Y. Setiady, E. K. Maloney, O. Ab, X. Zhang, J. Pinkas, T. A. Keating, R. Chari, H. K. Erickson, J. M. Lambert, Bioconjug. Chem. 2017, 28, 1371-1381.

[3] S. J. Walsh, J. D. Bargh, F. M. Dannheim, A. R. Hanby, H. Seki, A. J. Counsell, X. Ou, E. Fowler, N. Ashman, Y. Takada, A. Isidro-Llobet, J. S. Parker, J. S. Carroll, D. R. Spring, Chem. Soc. Rev. 2021, 50, 1305-1353.

[4] a) L. Conilh, G. Fournet, E. Fourmaux, A. Murcia, E.-L. Matera, B. Joseph, C. Dumontet, W. Viricel, Pharmaceuticals 2021, 14, 247; b) R. P. Lyon, T. D. Bovee, S. O. Doronina, P. J. Burke, J. H. Hunter, H. D. Neff-LaFord, M. Jonas, M. E. Anderson, J. R. Setter, P. D. Senter, Nat. Biotechnol. 2015, 33, 733-735; c) R. A. Kudirka, R. M. Barfield, J. M. McFarland, P. M. Drake, A. Carlson, S. Banas, W. Zmolek, A. W. Garofalo, D. Rabuka, ACS Med. Chem. Lett. 2016, 7, 994-998.

[5] a) T. Nakada, T. Masuda, H. Naito, M. Yoshida, S. Ashida, K. Morita, H. Miyazaki, Y. Kasuya, Y. Ogitani, J. Yamaguchi, Y. Abe, T. Honda, Bioorg. Med. Chem. Lett. 2016, 26, 1542-1545; b) T. Nakada, K. Sugihara, T. Jikoh, Y. Abe, T. Agatsuma, Chem. Pharm. Bull. 2019, 67, 173185; c) S. J. Keam, Drugs 2020, 80, 501-508.

[6] a) D. M. Goldenberg, T. M. Cardillo, S. V. Govindan, E. A. Rossi, R. M. Sharkey, Oncotarget 2015, 6, 22496-22512; b) J. M. Seligson, A. M. Patron, M. J. Berger, R. D. Harvey, N. D. Seligson, Ann. Pharmacother. 2021, 55, 921-931.

[7] D. V. Nadkarni, J. Lee, Q. Jiang, V. Patel, V. Sriskanda, K. Dutta, D. M. Meyer, Mol. Pharm. 2021, 18, 889-897.

[8] K. J. Pacholarz, P. E. Barran, EuPA Open Proteom. 2016, 11, 23-27.

[9] E. A. Hoyt, P. M. S. D. Cal, B. L. Oliveira, G. J. L. Bernardes, Nat. Rev. Chem. 2019, 3, 147-171.

[10] a) P. Strop, K. Delaria, D. Foletti, J. M. Witt, A. HasaMoreno, K. Poulsen, M. G. Casas, M. Dorywalska, S. Farias, A. Pios, V. Lui, R. Dushin, D. Zhou, T.

Navaratnam, T.-T. Tran, J. Sutton, K. C. Lindquist, B. Han, S.-H. Liu, D. L. Shelton, J. Pons, A. Rajpal, Nat. Biotechnol. 2015, 33, 694-696; b) P. Strop, S.-H. Liu, M. Dorywalska, K. Delaria, Russell G. Dushin, T.-T. Tran, W.H. Ho, S. Farias, Meritxell G. Casas, Y. Abdiche, D. Zhou, R. Chandrasekaran, C. Samain, C. Loo, A. Rossi, M. Rickert, S. Krimm, T. Wong, Sherman M. Chin, J. Yu, J. Dilley, J. Chaparro-Riggers, Gary F. Filzen, Christopher J. O'Donnell, F. Wang, Jeremy S. Myers, J. Pons, David L. Shelton, A. Rajpal, Chem. Biol. 2013, 20, 161-167.

[11] a) X. Li, T. Fang, G. J. Boons, Angew. Chem. Int. Ed. Engl. 2014, 53, 7179-7182; b) X. Li, T. Fang, G. J. Boons, Angew. Chem. 2014, 126, 7307-7310; c) R. van Geel, M. A. Wijdeven, R. Heesbeen, J. M. Verkade, A. A. Wasiel, S. S. van Berkel, F. L. van Delft, Bioconjug. Chem. 2015, 26,
2233-2242; d) T. B. Parsons, W. B. Struwe, J. Gault, K. Yamamoto, T. A. Taylor, R. Raj, K. Wals, S. Mohammed, C. V. Robinson, J. L. P. Benesch, B. G. Davis, Angew. Chem. Int. Ed. Engl. 2016, 55, 2361-2367; e) T. B. Parsons, W. B. Struwe, J. Gault, K. Yamamoto, T. A. Taylor, R. Raj, K. Wals, S. Mohammed, C. V. Robinson, J. L. P. Benesch, B. G. Davis, Angew. Chem. 2016, 128 2407-2413; f) S. Sun, P. Akkapeddi, M. C. Marques, N. Martinez-Saez, V. M. Torres, C. Cordeiro, O. Boutureira, G. J. L. Bernardes, Org. Biomol. Chem. 2019, 17, 20052012.

[12] R. P. Lyon, D. L. Meyer, J. R. Setter, P. D. Senter, Methods Enzymol. 2012, 502, 123-138.

[13] A. C. Stan, D. L. Radu, S. Casares, C. A. Bona, T. D. Brumeanu, Cancer. Res. 1999, 59, 115-121.

[14] Q. Zhou, J. E. Stefano, C. Manning, J. Kyazike, B. Chen D. A. Gianolio, A. Park, M. Busch, J. Bird, X. Zheng, H. Simonds-Mannes, J. Kim, R. C. Gregory, R. J. Miller, W. H. Brondyk, P. K. Dhal, C. Q. Pan, Bioconjug. Chem. 2014, 25, 510-520.

[15] a) L. Sun, T. Bulter, M. Alcalde, I. P. Petrounia, F. H. Arnold, ChemBioChem 2002, 3,731-783; b) L. Sun, I. P. Petrounia, M. Yagasaki, G. Bandara, F. H. Arnold, Protein Eng. 2001, 14, 699-704.

[16] a) I. S. Carrico, B. L. Carlson, C. R. Bertozzi, Nat. Chem Biol. 2007, 3, 321-322; b) P. Wu, W. Shui, B. L. Carlson, N. Hu, D. Rabuka, J. Lee, C. R. Bertozzi, Proc. Natl. Acad. Sci. U.S.A. 2009, 106, 3000-3005; c) J. E. Hudak, R. M. Barfield, G. W. de Hart, P. Grob, E. Nogales, C. R. Bertozzi, D. Rabuka, Angew. Chem. Int. Ed. Engl. 2012, 51, 4161-4165; d) J. E. Hudak, R. M. Barfield, G. W. de Hart, P. Grob, E. Nogales, C. R. Bertozzi, D. Rabuka, Angew. Chem. 2012, 124, 4237-4241; e) M. J. Appel, C. R. Bertozzi, ACS Chem. Biol. 2015, 10, 72-84.

[17] N. Janson, T. Krüger, L. Karsten, M. Boschanski, T. Dierks, K. M. Müller, N. Sewald, ChemBioChem 2020, 21, 3580-3593

[18] N. J. Agard, J. A. Prescher, C. R. Bertozzi, J. Am. Chem. Soc. 2004, 126, 15046-15047. 


\section{Entry for the Table of Contents}

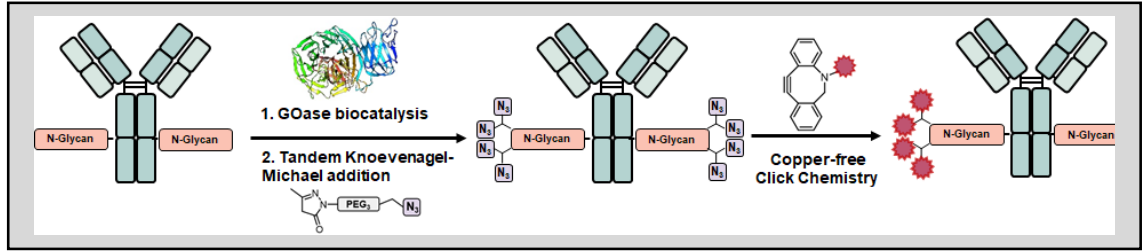

High-DAR antibody conjugates via Glycoengineering: The synthesis of antibody-drug conjugates with high drug loading from native antibodies are of great interest for development of biopharmaceuticals. Here we combine selective biocatalysis using galactose oxidase and Tandem Knoevenagel-Michael addition conjugation chemistry to achieve molecular DAR values of up to eight. 